\title{
On leveraged and inverse leveraged exchange traded funds
}

\author{
Rompotis, Gerasimos G.
}

- ReCeived: 28 november 2013

- ACCEPTED: 19 DeCEMBER 2013

\begin{abstract}
The leveraged and inverse ETFs are the subject of the current paper. At first, a comprehensive review of the literature on leveraged ETFs is provided. Then, the features, trading mechanism, and the advantages and disadvantages of leveraged ETFs are analyzed along with the similarities to and differences from the classic ETFs while tax considerations surrounding leveraged ETFs are highlighted too. Finally, the role of market volatility and its impact on the return of leveraged ETFs is accentuated.
\end{abstract}

Keywords:

ETFs, Seasonality, November effect, Performance, Risk, Tracking error.

JEL classification:

G11. 


\section{Fondos cotizados apalancados e inversos apalancados}

Rompotis, Gerasimos G.

\section{Resumen}

Los fondos cotizados apalancados e inversos constituyen el objeto de este artículo. En primer lugar se proporciona una amplia revisión de la literatura sobre la cuestión. Posteriormente se analizan las características, el mecanismo de trading, las ventajas y desventajas de los fondos cotizados apalancados junto con sus similitudes y diferencias con los fondos cotizados clásicos, y se hace hincapié en las consideraciones fiscales que los rodean. Finalmente, se puntualiza el papel de la volatilidad de mercado y su impacto en el rendimiento de los fondos cotizados apalancados. Además, este artículo proporciona una revisión de la literatura existente sobre los fondos cotizados apalancados e inversos.

\section{Palabras clave:}

Fondos cotizados, estacionalidad, efecto Noviembre, rendimiento, riesgo, tracking error. 


\section{Introduction}

The majority of the $1^{\text {st }}$ Generation's ETFs traded on U.S. and other stock exchanges worldwide aim at replicating the performance of a known index or benchmark either at the short-run or the long-run level. To do so, ETFs are usually fully invested in the securities of the underlying index, even though synthetic replication is also applicable. They are basically passively managed, which means that the synthesis of the managed portfolio usually changes only in response to changes in the components of the tracking benchmark.

The growing interest of investors in ETFs along with their need to apply more active investing strategies with ETFs gave birth to new more active types of ETF products, such as the leveraged and inverse leveraged ETFs, which are alternatively called bullish and bearish ETFs, respectively. The first leveraged and inverse ETFs were launched in the U.S. market by Proshares in June 2006 after being reviewed for almost three years by the Securities and Exchange Commission.

The leveraged ETFs are aimed at beating the underlying benchmarks and are designed to deliver twice or three times the performance of the benchmark over a pre-specified period (before fees and expenses), which usually does not exceed the one day. ${ }^{1}$ The inverse ETFs seek to short the market and provide performance opposite to various market benchmarks on a daily basis. In the case of inverse ETFs, there are those funds targeting to $-100 \%$ the return of the benchmark (inverse ETFs) as well as those funds aiming at $-200 \%$ or $-300 \%$ the benchmark's return (inverse leveraged ETFs). ${ }^{2}$ The stated multiples of 2:1 or 3:1 (in a positive or a negative way) can be treated as the targeted leverage ratio of funds.

The leveraged and inverse ETFs are the subject of the current paper. At first, the paper provides a comprehensive review of the existing literature on leveraged and inverse ETFs. Despite the short history of these ETF types, the relevant literature is sufficient mainly due to their increasing popularity with investors, expressed by the growth in assets under management by the vendors of leveraged ETF products, as well as the increasing criticism on them due to concerns about their complexity in addition to frequent instances of them failing to meet their daily investing targets. After the literature review, the focus of the paper is on the features, trading mechanism, and the advantages and disadvantages of leveraged ETFs are analyzed along with the similarities to and differences from the classic ETFs while tax considerations

\footnotetext{
I Albeit the existing leveraged ETFs have daily investment horizon, leveraged ETFs to track monthly returns are currently under development and no much time will elapse before they become a reality.

${ }^{2}$ For convenience purposes, we will be calling inverse and inverse leveraged ETFs as inverse ETFs because their trading mechanisms are essentially similar to each other with the additional feature that the latter are designed for multiple returns.
} 
surrounding leveraged ETFs are highlighted too. Finally, the role of market volatility and its impact on the return of leveraged ETFs is accentuated.

\section{Literature Review}

Along with the growing popularity of leveraged and inverse ETFs with investors expressed by the growth in their assets and trading activity (leveraged ETFs are among the most tradable securities), ${ }^{3}$ they have also been of great interest to researchers. The main issues examined by the financial literature concern the ability of leveraged ETFs to deliver their daily targets and the dynamics that affect their pricing behavior, such as their structure, the required rebalancing of leveraged ETF portfolios and the effects of market volatility on leveraged ETFs' performance. Moreover, the long-term return of leveraged ETFs and the consequent suitability of these tools to investors having wider investment horizons have been considered by researchers. Special attention has also been paid to the performance of leveraged ETFs compared to regular single-beta ETFs having common benchmarks. The impacts of leveraged ETFs on the volatility of their underlying stocks have been studied too. Other issues including the pricing efficiency in terms of premiums/discounts between the trading values of leveraged ETFs and their NAV have also been examined.

\subsection{Meeting daily and long-term targets}

On the ability of leveraged ETFs to achieve their daily investing targets, Trainor and Baryla (2008) report that, on average, leveraged ETFs do meet their daily targets. However, by examining longer periods, they find that the long-run returns of leveraged ETFs are not comparable to two or three times the return of benchmarks due to the constant leverage trap and the lognormal nature of continuously compounded returns. The authors also point out that the effect of compounded returns becomes more intense as holding period increases. Despite the inability of leveraged ETFs to achieve their stated multiples over longer periods, in the long-run, they expose investors to volatility which equals the standard deviation of the index multiplied by the leverage ratio.

Mackintosh (2008) reports that the long-run performance of leveraged ETFs is not linearly related to the return of indices and shows that it is quite different from the index's return multiplied by the leverage ratio. The author attributes this discrepancy to the daily dynamic re-leveraging of the ETF portfolio required for maintaining constant the desired exposure to the tracking index. In addition, he reveals that there

${ }^{3}$ Charupat and Miu (20II). 
is a direct relationship between the level of tracking error (as measures by the difference in returns between leveraged ETFs and indices) and market volatility. This means, that the higher volatile the market, the higher the difference between the longterm return of leveraged ETFs and the stated multiples.

Hougan (2009) examines how long a leveraged ETF should be held. He finds that the daily returns of leveraged ETFs do not abstain from the targets and the tracking errors observed are insignificant. Tracking errors greaten as the investment horizon widens. However, the weekly returns of leveraged ETFs still present a good fit with their stated multiple. Returns start to significantly deviate from the return targets when monthly or longer performance is assessed.

Cheng and Madhavan (2009) accentuate a path-dependence pattern in leveraged ETFs' returns, which can decay the wealth obtained by a buy-and-hold investor, who will treat these products as long-term investment vehicles. In this respect, the high magnitude of costs relating to management fees and bid-ask spreads as well as the tax inefficiency of leveraged ETFs due to the high portfolio turnover and the potential large distributions of capital gains are highlighted as the factors that contribute to the erosion of returns received by long-term investors using leveraged ETFs.

Murphy and Wright (2010) investigate the performance of twelve commodity-based leveraged ETFs. They find that these ETFs do manage to deliver their stated multiple in the short-run. However, in the long-run, these ETFs basically fail to perform as they are expected even though they reveal evidence that some of their sample's funds performed better than their stated multiples in the long-run. The authors conclude by recommending against using leveraged ETFs in any sort of buy-and-hold strategies.

Rompotis (2011) investigates whether the inverse leveraged ETFs are capable of achieving their daily wagers and computes an average deviation from the daily target of $-0.034 \%$, which is quite low and indicates a satisfactory fit between the daily returns of inverse ETFs and their targets. Moreover, the author reports that for about the $62 \%$ of the examined trading days the absolute difference between the return of short ETFs and their targets does not exceed the 50 b.p. This finding supports the inference about the sufficiency of short ETFs in meeting their daily targets. In the analysis of daily performance, he finds that there is an inverse relationship between the current and the one-day lagged returns. On the question of whether short ETFs are suitable to longterm investors, Rompotis (2011) finds that these products do not produce any significant alpha and, consequently, they should not be used in buy-and-hold strategies.

Charupat and Miu (2011) compare the returns of Canadian leveraged ETFs to their daily targets over periods exceeding one-day and find that for one-week holding 
periods the tracking errors, namely the difference between the accumulated return of ETFs and the corresponding target returns (i.e. the index return multiplied by the leverage ratio), are quite small. Tracking errors surge as the holding periods extend.

Shum (2011) also investigates the performance of Canadian leveraged ETFs. She first finds that the performance of these ETFs abstain from their stated multiple for holding periods that exceed one month. The magnitude of deviation increases as the duration of the holding period lengthens. Moreover, she finds that the bull ETFs are more capable of delivering their target in the long-run than their bear counterparts. Finally, in addition to the influence of compounding of leveraged ETFs' returns on their ability to perform in line with their daily targets in the long-run, she accentuates the pervasive role of managers' inability to efficiently replicate the benchmarks along with pricing inefficiencies in leveraged ETF market, and especially during the financial crisis of 2008.

Rompotis (2012) studies the return behavior of leveraged and inverse leveraged Proshares, which are the industry's leaders. He finds that there is a maximum absolute gap between the return of short and long Proshares not exceeding the 50 b.p. on about the $57 \%$ and $53 \%$ of trading days, respectively. Moreover, he searches for any material day-of-the week effects on performance finding that the short ETFs present their best replication efficiency (with respect to target returns) on Wednesday.

In a revised study on Proshares, Rompotis (2013) reconfirms the sufficiency of these ETFs to deliver their daily goals. In particular, he reports that, when NAV returns are considered, the short and long Proshares perform at an acceptable level (i.e. the difference from the daily target does not exceed the 50 b.p.) in about the $85 \%$ and $82 \%$ of trading days, respectively. The percentages are worse when returns are calculated in trading prices terms $(63 \%$ for inverse Proshares and $61 \%$ for the leveraged ones).

On the long-term performance of leveraged ETFs, Hill and Foster (2009) show that the leveraged and inverse ETFs can be successfully used in more long-run investing strategies. They show that the impact of compounding on these ETFs over multiday periods is not material and, therefore, the probability of approximating the one-day target for beyond one day periods is high. What is crucial in this respect is the length of the period, which should be relevantly short. In addition, the market volatility is needed to be low.

Lu et al. (2012) demonstrate that over holding periods not exceeding one month investors can safely assume that the leveraged (inverse) ETFs would provide twice the return (twice the negative return) of the tracking indices. For longer periods, the performance of leveraged ETFs can differ from the return of indices multiplied by the 
leverage ratio. According to the authors, in the case of inverse ETFs, the deviation occur over quarterly periods while for leveraged ETFs the deviation occurs for holding periods up to one year.

\subsection{Significance of rebalancing}

Hill and Foster (2009) note that rebalancing the ETF portfolio is crucial in order for the stated multiple to be achieved over longer periods. According to the authors, rebalancing is an effective tool for investors whose goal is to approximate the daily leverage target over longer time intervals. They suggest a straightforward process which involves monitoring the returns of the tracking index versus the return of ETFs and establishing a trigger percentage of deviation as a basis for the rebalancing strategy or prescribing a calendar trigger on a weekly, monthly or other time basis. They also point out that investors should set an appropriate trigger for rebalancing by taking into account the volatility of the index. They conclude that compared to returns from an un-rebalanced fund, rebalancing reduces the size of the gap between the ETF's return and the target so that the return received by investors over multiday periods is closer to the daily multiple of the index.

The importance of rebalancing for achieving the daily target over longer periods is highlighted by Hill and Teller (2009), too. They perform a historical analysis of staticleveraged versus rebalanced returns for a variety of indices across a range of market conditions and provide evidence of the effectiveness of rebalancing over six-month investing horizons. The frequency of rebalancing depends on the volatility of the underlying index and the structure of fund, namely, on whether it is a leveraged or an inverse leveraged fund. In particular, the authors report that for long funds levered on low volatile indices, the necessary rebalancing frequency ranges from three to four times a year. On the other hand, the short funds tracking highly volatile indices need to be rebalanced on a weekly basis.

Carver (2009) in his turn acknowledges the significance of rebalancing. He indicates that that the leveraged ETFs can perform poorly over longer time horizons, even when the underlying index performs well, if efficient rebalancing of funds' positions is not performed in combination with the geometric nature of returns compounding. In fact, he shows that highly leveraged ETFs are likely to converge to zero over longer time horizons, especially during extreme market conditions. However, under moderate market conditions, ETFs implementing adaptive leverage policies might produce more attractive results over longer time horizons.

The influence of rebalancing is also examined by Avellaneda and Zhang (2010), who compare the performance of funds which are frequently rebalanced to the simple 
leveraged buy-and-hold strategy. The authors find that the frequently rebalanced funds tend to underperform the static leveraged portfolios and this underperformance will be higher in periods of high volatility because during such periods the daily re-leveraging results in higher portfolio turnover. The leveraged ETFs outperform the static strategy only when returns are high and volatility is low. However, the authors conclude that leveraged ETFs can be used to replicate a pre-defined multiple of the underlying index returns over longer periods provided that dynamic rebalancing strategies are performed.

Charupat and Miu (2011) also comment on the impact of rebalancing on the long-term performance of leveraged ETFs in combination with market volatility. More specifically, they state that the frequent rebalancing of leveraged ETF portfolios over high volatility periods in order for the required leveraged exposure to the tracking index to be maintained is the culprit for the discrepancy between the long-term returns of ETFs and their corresponding multiples. The authors conclude that in order for investors to avoid such tracking errors, they should limit the duration of their holding periods.

Dulaney et al. (2012) investigate the impact of rebalancing's frequency on the tracking error of leveraged ETFs, namely the difference between their return and the stated multiple. They consider three different frequencies; daily, weekly and monthly. They conclude that the decrease in the frequency of rebalancing, e.g. the switch from daily to weekly adjustment of the leveraged ETF portfolio, can substantially decrease tracking error.

\subsection{Investors in leveraged ETFs}

On the type of investors using leveraged ETFs, Cheng and Madhavan (2009), report that these funds are mainly used by short-term traders but they have also started appealing individual investors seeking to hedge their positions or add leverage to their portfolios. Furthermore, they investigate the dynamics affecting the return behavior of leveraged and inverse ETFs and their impact on market volatility and liquidity showing that the daily rebalancing of these funds can induce volatility and trading volume towards the close of a trading day.

Rollenhagen (2009) stresses that the choice on investing or not in leveraged ETFs depends on the risk/performance profile of each individual investor. He adds that in order for an investor to expect to return twice or three times the return of an asset, they must tolerate the respective uptick in risk. Therefore, the leveraged ETFs are not suitable to everyone but only to those investors who are experienced and skilled enough to understand the mechanics and the hazards entailed by trading with leveraged ETFs.

Wang (2009) suggests that the activity of "noise traders", and especially during periods of high volatility, drives the prices of leveraged ETFs away their fair value in a short term 
interval. This effect leads to market inefficiency. Moreover, the increased demand of investors for leverage and hedging can induce the assets held by leveraged ETFs and the respective rebalancing action. Quite often, the high demand for leveraged ETF shares is manifested near the close of trading day. The consequent rebalancing might distort the market price of leveraged ETFs and create additional inefficiency.

Guedj et al. (2010) measures the investing holding periods of leveraged ETFs. They find that, even though these ETFs are by construction suitable to investors having very short investing horizons, a considerable fraction of investors hold leveraged ETF shares for periods exceeding even one quarter. In this context, they add that the daily turnover of leveraged ETF portfolios is inversely related to the holding period.

Trainor (2011a) acknowledges the compounding issue affecting the long-term performance of daily rebalanced leveraged ETFs and examines their suitability to longer-term investors vis-à-vis funds being rebalanced on a monthly basis. ${ }^{4} \mathrm{He}$ finds that for a three-month period, the latter are more advantageous to investors having more long-term horizon than the leveraged ETFs, which adjust their exposure to the underlying assets on a daily basis. Going further, the author notes that the superiority of monthly leveraged funds over their daily peers for relevantly long-tern periods depends on the market trend and volatility. In particular, he reports that over a period with high positive market trend (or high negative trend in the case of inverse leveraged funds) coupled with low volatility, the monthly rebalanced funds outperform their daily peers. Nevertheless, the author emphasizes that even the monthly leveraged funds are not suitable to the typical buy-and-hold investors. On the contrary, Trainor (2011b) reports that the long-term investors can hold leveraged ETFs for long periods and gain returns even greater than the daily multiple implies providing that they avoid to maintain their positions in leveraged ETFs during high volatile market periods.

Charupat and Miu (2011) examine the type of investors trading leveraged ETFs along with the pricing behavior of these tools employing data from the Canadian ETF market. They find that the leveraged ETFs (and especially those with positive multiples) are more preferable to retail traders having very short investing horizons not exceeding 15 days, who want to implement specific trading strategies.

With respect to the impact of investors' horizon on the return of leveraged ETFs, Leung and Santoli (2012) report that performance generally declines as the duration of holding

\footnotetext{
${ }^{4}$ It should be that at the time this article was being written, there were no monthly leveraged ETFs available but only mutual funds and Exchange Traded Notes (ETNs). ETNs have different structure from ETFs. In particular, ETNs are issued as senior debt notes whereas there are also differences in their tax treatment and the risks involved with trading each of these alternative products. For a more detailed analysis of the differences between ETFs and ETNs refer to the following link: http://www.investopedia.com/ articles/06/etnvsetf.asp\#axzz2KObh7lqQ
} 
period increases. Therefore, investors having long-term horizon should probably avoid embarking on leveraged ETFs. They also reveal that the impact of horizon is more severe in the case of highly leveraged ETFs. Furthermore, the authors explore the effect of leverage on the risk involved in trading with leveraged ETFs by introducing the idea of an admissible range of leverage ratios, which examines the leverage ratios for which a leveraged ETF satisfies a predefined risk constraint. A mathematic analysis is provided in this respect, which aims at helping investors form their investing decisions according to their tolerance to risk by excluding the leveraged ETFs that will be deemed over risky.

Curcio et al. (2012) emphasize that naïve buy-and-hold investors can lose value when using leveraged ETFs to invest in the real estate sector. Nevertheless, comparing the advantages and risks of leveraged ETFs, the authors conclude that the tracking error associated with holding leveraged ETFs beyond a single trading day should not prevent investment managers from using these ETFs in real estate portfolios. The reasoning is that leveraged ETFs are useful hedging instruments that can enhance returns and, under certain circumstances and frequent monitoring, they can be judiciously employed for longer than a single day by sophisticated traders and well-informed portfolio managers.

Dulaney et al. (2012) report that a large fraction of investors seem not to fully comprehend the mechanisms, risks and the appropriate usages of leveraged and inverse ETFs. As a corollary, they hold these products for much longer than a day exposing themselves to high risk and experiencing great losses. The authors partially attribute this inability of investors to the ambiguous disclosures about the investing objectives of these products made by leveraged ETF providers in funds' prospectuses and highlight the necessity of more comprehensible disclosure notes that will enable investors to fully appreciate the risks deriving from holding longterm leveraged ETFs.

\subsection{Leveraged ETFs versus traditional instruments}

Murphy and Wright (2010) investigate the long-term return of twelve leveraged Proshares tracking commodities or commodity-based indices vis-à-vis the underlying commodities or indices. They find that ten out of these twelve products underperform their corresponding unleveraged commodities or indices over their entire trading life. The authors attribute the underperformance of leveraged ETFs to the price volatility of the underlying commodities and indices. In particular, a movement of underlying assets to the wrong direction will harm the long-term return of leveraged ETFs.

Guedj et al. (2010) compare the longer-term return of leveraged ETFs to the respective returns of their benchmarks during comparable holding periods. They find that the 
leveraged ETFs underperform the tracking indices in any holding period considered. They also find that the longer the holding period, the greater the investment shortfall from the benchmark.

Barnhorst and Cocozza (2011) examine the long-term return of one bull and one bear ETF compared to performance of one regular ETF peer. The three funds track the same market index, which is the Dow Jones U.S. Oil and Gas Index. They find that over a period of five months (December 2008-April 2009) the leveraged ETFs underperform the corresponding single-beta regular ETF. Based on the results, the authors recommend against usage of leveraged ETFs by traditional unsophisticated investors. In contrast, investors seeking just to lever their market exposures without needing constant trade monitoring and complicated strategies, should consider the employment of Exchange Traded Notes and futures contracts.

Rompotis (2011) studies the main trading features of inverse leveraged ETFs compared to corresponding regular ETFs tracking the same indices. The author reports that the short ETFs are significantly more expensive than traditional peers, they present lower trading activity as expressed by trading volume and frequency (i.e. days with no zero volume), are more volatile intraday and exhibit higher tracking errors with respect to their return targets. Moreover, by comparing these alternative investing vehicles from a long-run perspective, he finds that, both the average daily and the accumulated return of short ETFs is inferior to the corresponding returns of traditional ETF counterparts. In addition, the short ETFs are found to be more risky. When comparing the individual daily returns of leveraged and regular ETFs, Rompotis (2011) reveals that the latter beat the former over above the $50 \%$ of trading days.

Charupat and Miu (2011) compare the Canadian leveraged ETFs to their single-beta peers showing that the former have much shorter holding periods than the latter and lower values per trade. In addition, the leveraged ETFs are more active than the regular ones in terms of trading activity. Furthermore, the authors find that the average differences between the trading prices of leveraged ETFs and their NAV are not that sizeable. However, they point out that large premiums and discounts are likely to occur and that the premiums/discounts of leveraged ETFs are more volatile than those of regular ETFs due to the embedded leverage.

Dulaney et al. (2012) compare the return received by investors in leveraged ETFs holding these products for longer than one day periods to the return of the underlying indices as well as the return gained via a "margin account". ${ }^{5}$ They find that the ETFs underperform the indices and the corresponding investments in derivatives in any

\footnotetext{
${ }^{5}$ The term "margin account" refers to investing in similar underlying assets via futures contracts.
} 
holding period longer than a day. In addition, they find that the deviation between returns grows as the holding period increases.

\subsection{Effects of leveraged ETFs on underlying markets}

Cheng and Madhavan (2009) built a model to examine the impact of rebalancing trades of leveraged ETFs performed towards the close of trading day on market volatility. They base their analysis on market-on-close volume (i.e. trades specified to be executed at the market close price) and find that the trading activity of leveraged ETFs at the end of the day induces the volatility of the respective market indices.

Trainor (2010) seeks to answer whether the trading activity of leveraged ETFs during the afternoon and close to the end of the trading day contributes to the increase in the volatility of the tracking indices. His study focuses on the possible effects caused by leveraged ETFs in stock prices during the financial crisis of 2008. The author refutes the accusations on leveraged ETFs' rebalancing trades as being the culprit for the increased volatility those days by stating that the increased volatility of the S\&P 500 Index is just a spurious coincidence. More specifically, market volatility towards the end of day seems to increase but this is also the case for volatility throughout the entire trading day. Therefore, market volatility cannot be driven by the rebalancing actions of leveraged ETFs at the end of day.

Blau and Brough (2011) examine the hypothesis whether the trading activity of inverse ETFs convey any message about the future prices of the underlying indices. They reach two main inferences. The first one is that the trading volumes of bear ETFs increase after periods of negative pricing fluctuations of the tracking benchmarks. This pattern implies that investors in bear ETFs are momentum traders. The second finding is that the trading activity of inverse ETFs does not enable any prediction about the forthcoming movements of indices. In combination, the two findings demonstrate that there is no meaningful information in the trading of bear ETFs.

Charupat and Miu (2011) study how the deviation between the trading and net asset values of Canadian leveraged ETFs may affect the return of the underlying indices. The authors find that the premium in trading prices of bull leveraged ETFs are inversely related to the return of benchmarks. The opposite relationship applies to bear ETFs. The authors interpret the correlation of bull and bear ETFs with indices' returns as evidence of the provoking impact of leveraged ETFs' end-of-day rebalancing trades on the increased volatility of the underlying markets.

Welker (2012) investigates the effects of leveraged and inverse ETFs on the pricing behavior of the underlying indices throughout the day following the introduction of 
these ETFs. The results obtained are mixed. In particular, the author finds that there are cases where the introduction of a leveraged ETF was accompanied by increased volatility and trading volume for the underlying stocks the next day. Yet, there are also cases where the launch of a leveraged ETF coincided with a drop of underlying stocks' price fluctuation and volume the next day. Therefore, there is not a uniform inference to reach about the impact of leveraged ETFs on the tracking assets' price behavior.

Bai et al. (2012) also examine the impact of leveraged ETFs' late trading (i.e. towards the close of trading day) on the stocks belonging to real estate sector. They find that the stock components experience increased return variance and trading volume late in the day. The impact is greater in the case of smaller and less liquid stocks. They attribute this increased trading activity to the rebalancing trades of the corresponding leveraged ETFs tracking the Dow Jones US Real Estate Index. They also find that the greater the level of rebalancing activity, the greater the variance in the prices of stocks. Finally, evidence is provided that the positive relationship between the rebalancing of leveraged ETFs and stocks' late returns is reversed at the beginning of the next day.

Curcio et al. (2012) investigates the impact of leveraged ETFs' introduction on the prices of real estate stocks too. By comparing the volatility of stocks over 64 trading days before and after the inception of leveraged ETFs, they find that volatility of real estate stocks has dramatically increased after the launch of leveraged ETFs. They also note that the highest volatility accrued around the initiation of these ETFs.

Haryanto et al. (2012) study the influence caused by the rebalancing activity of leveraged ETFs towards the close of trading day on the price volatility of the underlying stock shares. The main finding is that rebalancing does affect the end-of-day market volatility although the impact is more evident and economically meaningful during days with high price swings of stocks before the close of trading day (i.e. price movements up to $3.30 \mathrm{pm}$ ) when the rebalancing trades are likely to begin. The impact of leveraged ETFs on stocks' volatility is linked to actions by leveraged ETFs' swap counterparties towards the close of the day, which need to hedge their positions to the total return swap and execute trading orders that may induce the end-of-day volume and volatility of the underlying stocks.

Chen and Diaz (2012) investigate the interrelationship between leveraged and inverse leveraged ETFs and their underlying indices. More specifically, they examine whether there are any spillover effects on returns and volatilities caused by these ETFs to their indices and vice versa. The authors reveal a bilateral relationship of spillover effects of returns and volatilities. In particular, they find that the lagged returns of leveraged ETFs affect the current returns of indices in a positive way. The opposite trend is found in the case of inverse leveraged ETFs. On the other hand, the lagged returns of indices 
are negatively related to the current returns of leveraged ETFs as a corollary of increasing the exposure to tracking indices with the usage of additional swaps. In the case of inverse ETFs, the lagged returns of benchmarks positively affect the performance of these ETFs the following day.

\section{Features and usages of leveraged ETFs}

Leveraged and inverse ETFs offer investors exposure to various markets, either small, mid- or large-caps, domestic or foreign, and broad or sector. In addition, they offer exposure to several asset classes such as equities, bonds, commodities, precious metals and currencies. For instance, Nasdaq-100 Index, Dow Jones Industrial Average and Standard and Poor's 500 Index are massively used benchmarks by leveraged and inverse ETFs. Sector indices such as those included in the Dow Jones family of sector indices investing in stocks of firms belonging to financial services sector, oil and gas sector, basic materials sector, healthcare sector, industrial sector and other are also tracked by leveraged and inverse ETFs. Some of the MSCI country or regional indices (e.g. MSCI Japan Index or MSCI Emerging Markets Index) and FTSE country indices (e.g. FTSE/Xinhua China 25 Index) are betted by leveraged and inverse ETFs. Finally, there are leveraged ETFs written on gold and silver, U.S. Dollar/Japanese Yen exchange rate, as well as leveraged ETFs written on treasury and other bond indices.

Leveraged ETFs aim at outperforming their benchmark on a daily basis and in order to do so, they are designed to include the securities in the index, but also include derivatives of the index's securities and the index itself, which are agreements that provide exposure to the desired index without needing a full replication of it. ${ }^{6}$ These derivatives include options, forwards, swaps and futures. They can also use borrowed capital so as to magnify the return of the underlying benchmarks. Leveraged ETFs resorting to debt aim at future profits from the investments they finance via indebtedness which will exceed the cost of borrowed capital. Inverse ETFs use both swaps and futures but swaps predominate by a wide margin because swaps are more flexible than futures as futures require standard amounts and times to expiration. As reported by Elston and Choi (2009), the inverse ETFs using swaps promise to pay a fixed amount and they will receive an amount depending on the return of the selected benchmark. On days of market recession, the counterparty's payments increase. ${ }^{7}$ On the other hand, the counterparty seeks to hedge its risk by shorting the stocks comprising the index.

\footnotetext{
${ }^{6}$ For instance, the leveraged ETFs offered by Direxion Shares generate between $10 \%$ and $100 \%$ of the requisite index exposure from equities and the rest from derivatives while the inverse leveraged ETFs offered by this vendor generate their full exposure through derivatives. Refer to the following link: www.direxionfunds.com/wpcontent/uploads/2012/09/Understanding_Exchange_Traded_ Funds.pdf $\square$

${ }^{7}$ Elston and Choi (2009) refer that usually the counterparty is a large swap bank such as Goldman Sachs.
} 
When it comes to return of leveraged ETFs, it should be noted that, performance is eroded by fees and expenses incurred by the fund. Leveraged ETFs incur three types of expenses. The first one regards the fees charged by the ETF's management company for the provision of advisory and other services along with costs relating to marketing and other third parties fees. The management fee is calculated as a fixed percentage on the ETF's assets every year and accrued on daily basis. The second type of cost regards the accrued financial cost. Financial cost concerns either the interests paid on the borrowed capital for the implementation of leverage or the cost of holding derivatives, which all have an interest rate built into their pricing. This rate is the riskfree rate and approximates the interest rates of country sovereign bonds. The last source of costs relates to the transactions costs incurred when buying and selling the derivatives or stocks used for the achievement of daily investments goals. It must be noted that even though the interest and transaction costs are not always easy to identify and measure, they do erode the return of leveraged ETFs and their ability to deliver their goals.

As far as the usages of leveraged ETFs are concerned, it should firstly be noted that they are an alternative and simple way for investors to deploy leverage in their portfolios without needing to use derivatives or execute multiple trades. Furthermore, the leveraged ETFs are usually assumed to achieve their daily goals and, thus, they are a good way of realizing short-term market gains. As a consequence, they are mainly addressed to short-term investors seeking to take advantage of the current trends in stock market and realize short-term gains, particularly intraday. In addition, the inverse ETFs allow investors to hedge long positions in their portfolios without having to go short any securities. ${ }^{8}$ In this respect, active traders can use the inverse ETFs as a substitute for short-selling the underlying asset when this asset is difficult to borrow. ${ }^{9}$ Moreover, the leveraged ETFs offer investors the chance to speculate on the direction of broad domestic or foreign market indices, oil and gas prices, gold and other basic materials, agricultural commodities, currencies and more.

\section{Risks associated with leveraged ETFs}

On the question of risks associated with investing in leveraged ETFs, there are certain considerations that must be taken by investors. In particular, the most important risk relates to the impact of market volatility on the performance of leveraged ETFs and their efficiency in meeting their daily targets. In general, leveraged ETFs are very sensitive to market volatility and during periods of significant fluctuation in equity markets, the prob-

\footnotetext{
${ }^{8}$ Refer to Hill and Teller (2010) for a detailed analysis of hedging strategies with leveraged ETFs.

9 Avellaneda and Zhang (2010).
} 
ability of leveraged ETFs failing to deliver their goals is increased. Therefore, the more volatile is the market, the less likely are investors to receive the expected return. In addition, in a highly volatile investing environment, the longer investors hold leveraged ETFs (either long or shorts), the further away can EIFs get from achieving their target to double or triple the performance of the tracking index (in a positive or a negative way). ${ }^{10}$

The second kind of risk relates to the variance in the market prices of leveraged ETFs. More specifically, given that the shares of leveraged ETFs are traded on the secondary market, their market price will fluctuate in response to changes in their NAV but they will also be affected by supply and demand forces. It is not easy to predict whether a leveraged ETF will trade at a premium or a discount to its NAV and, thus, an investor could obtain shares of a leveraged ETF whose market value is somehow inflated in comparison to the value of the underlying holdings and vice versa.

The last source of significant jeopardy regards the so-called "counterparty risk", which is incurred due to the employment of derivatives, such as swap agreements, in order for the desired index exposure to be attained. In this agreement, the one party will make payments based on a pre-determined interest rate, which can be either fixed or variable. The other party of the agreement will make payments according to the total return of the underlying asset or index. The counterparty risk relates to a possible monetary loss a leveraged ETF could be exposed to due to difficulties encountered by the counterparty in meeting its obligations prescribed by the relevant contract.

There are several possible ways to deal with counterparty risk, which are described in Bush (2009). At first, it can be mitigated by diversifying among the co-operating brokers acting as counterparties to the leveraged ETF as well as assessing their financial wealth and credit quality (through monitoring their financial statements or via any other appropriate way) and terminating co-operation with those organizations signaling material signs of insolvency. It should be noted however that, according to Bush (2009), in the case of leveraged ETFs having low assets the diversification via co-operating with several counterparties may not be affordable.

Going further, a leveraged ETF may decide to enter into very short-term swap agreements, which can expire next day or in a thirty-day time. Compared to swaps having one-year life span, the short-term ones lowers the exposure of leveraged ETF to its counterparty. The usage of futures instead of swaps is also suggested as a way of mitigating counterparty risk. According to Bush (2009), however, the advantage of futures over swaps in alleviating counterparty risk can be off-set by their tendency to

${ }^{10}$ The role of volatility and its effects on the performance of leveraged ETFs are comprehensively described in a subsequent section of this paper. 
exacerbate the failure of leveraged ETFs to meet their daily targets. On the contrary, a swap enables the leveraged ETF to control more efficiently the targeted performance.

A last tool to deal with counterparty risk suggested by Bush (2009) involves a tri-party agreement among the leveraged ETF, a swap provider and a custodian. In this agreement, the collateral needed is segregated into an account with the custodian. In an ordinary swap agreement between the ETF and the swap provider, the collateral would be held by the swap provider. However, with the tri-party contract, in case the swap provider defaults suddenly or less suddenly, the collateral would not be at risk and, thus, the counterparty risk would be diminished. Nevertheless, it should also be noted that the latter type of agreement carries a higher cost than those incurred between a leveraged product and the swap provider.

\section{Trading mechanism and performance of leveraged ETFs}

\subsection{Management of leveraged ETFs}

The leveraged ETFs work by doubling or tripling in a positive or a negative way an investment in a certain asset such as a stock index, bond index, commodity, precious metal or currency exchange rate. Asset managers such as banks and ETF companies use financial engineering to create funds to replicate the asset but also to provide additional return compared to the return of the assets. Figures 2.1 and 2.2 found in Avellaneda and Zhang (2010) constitute a schematic presentation of the management of a leveraged (bullish/long) ETF and an inverse (bearish/short) ETF.

Figure 1 shows the existence of the authorized participants, which typically are large institutions acting as market makers or specialists. The authorized participants determine the number of the leveraged ETF shares outstanding on a daily basis. Based on the calculations of authorized participants, the manager of the leveraged ETF finds out how many shares have been created or redeemed and accordingly adjusts the exposure to the underlying asset using "dynamic" leverage. ${ }^{11}$

The adjustment of exposure takes place on a daily basis. In the example shown in Figure 1, a two-beta long ETF is considered. The leverage or financial engineering employed by the ETF manager consists in borrowing an amount from the money market (e.g. \$100) and doubling the exposure to the asset (i.e. \$200) with the usage of securities comprising the underlying asset and derivatives on these securities or the

\footnotetext{
"Dynamic leverage is the opposite of the static leverage, which means levering an investment position one and "letting it ride". Dynamic leverage entails daily rebalancing and adjustment of the position. These definitions of static and dynamic leverage have been found in Little (2010).
} 
asset itself. The borrowing and investments are daily activities performed by the long ETF manager in order for the desired level of exposure to the asset to be maintained.

\section{Figure 1. The management of a leveraged (bullish/long) ETF}

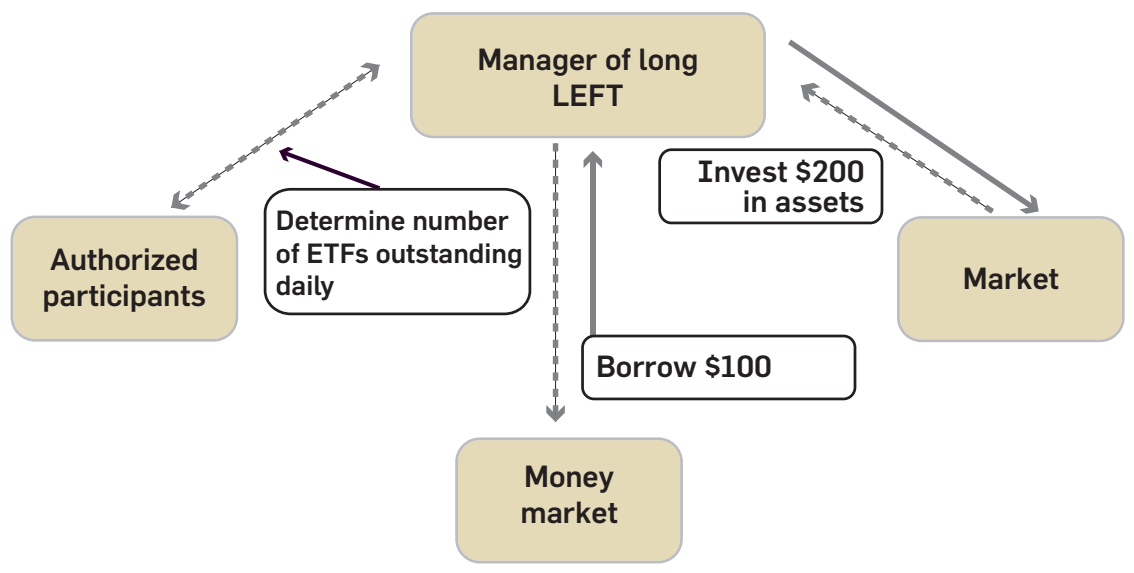

SOURCE: AVELLANEDA AND ZHANG (2010).

Figure 2 presents the management mechanism of a double-inverse ETF. Again, there are the authorized participants, which define the number of the short ETF's shares outstanding on each trading day and inform the manager of the short ETF in this respect. The manager adjusts the exposure of the short ETF daily by going short the underlying asset with double leverage and investing the proceeds in the money market.

As inferred from the description of long and short ETFs' management, one key point in the operating mechanism of leveraged and inverse ETFs is the fact that the leverage applies only on a daily basis. Unless the exposure of ETF to the underlying asset is rebalanced at the end of each trading day, the degree of leverage changes over time in response to changes in the prices of the underlying assets. In the case of long ETFs, leverage ratio increases as the value of the underlying securities increase and vice versa. The opposite trend applies to short ETFs. Therefore, the continuous rebalancing is crucial in order for leveraged ETFs to achieve their investment goals on a daily basis.

On a profitable day, a long ETF is under-leveraged for the following day and needs to increase its index exposure by buying more stocks or derivative products on the index. On a recessing day, this ETF is over-leveraged and needs to reduce its exposure to the underlying index by selling stocks or derivatives. On the other hand, the daily rebalancing of a leveraged short ETF requires from it to lower its exposure to the tracking asset if the market goes up and increase its position if the market goes down. In the case of short ETFs, the increase in index exposure is achieved via shorting or selling securities in the index. 
Figure 2. The management of an inverse leveraged (bearish/short) ETF

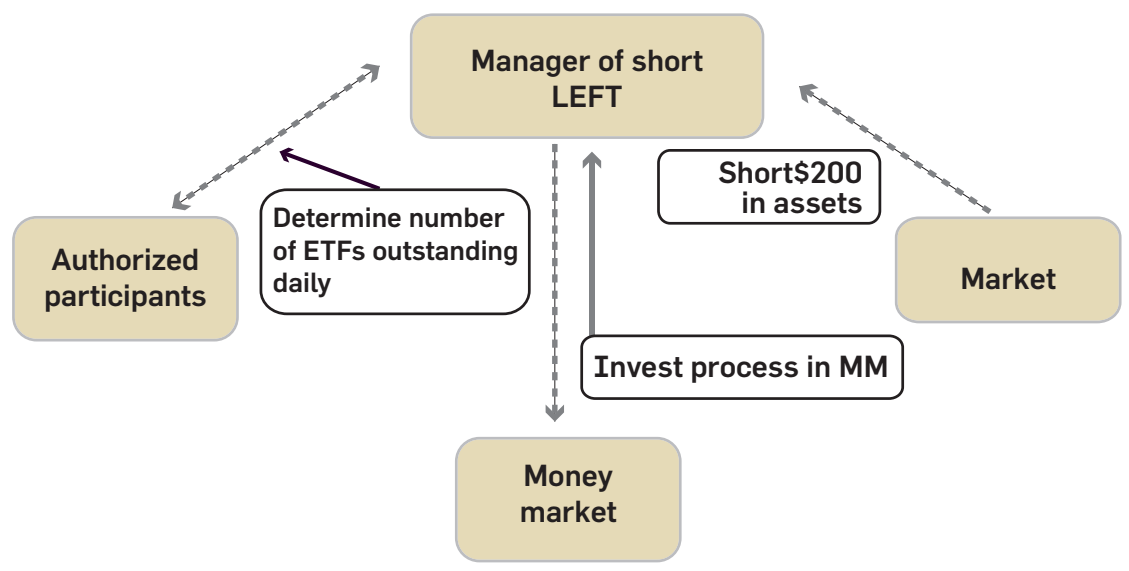

SOURCE: AVELLANEDA AND ZHANG (2010).

According to Proshares, there are two common approaches to implementing a rebalancing strategy. ${ }^{12}$ The first approach is "trigger-based" and the second is "calendar-based". The trigger-based approach activates rebalancing each time the difference between the return of the underlying and ETF's return reaches a specific threshold (e.g. $\pm 5 \%$ or $\pm 10 \%$ ). The calendar-based approach prescribes rebalancing at pre-defined intervals (e.g. weekly, monthly or quarterly). The main difference between the two approaches is that the calendar-based strategies are generally less tuned to market conditions than the trigger-based strategies. This means that they can respond to volatile market conditions in some delay. In addition, these strategies require more trading during periods of low market volatility. Another drawback of calendar rebalancing is that it ignores the size of the divergence between the index return and the return of ETF, the careful monitoring of which is absolutely necessary.

The frequency of rebalancing can be affected by several factors relating to the features of ETFs and benchmark as well as market conditions. One significant factor influencing the rebalancing process is the leverage ratio of ETF. The higher the ETF's multiple, the more frequent rebalancing is needed. Therefore, a triple-leveraged ETF requires more frequent rebalancing than a double-leveraged fund. Moreover, an inverse-leveraged ETF needs more rebalancing compared to a respective leveraged ETF because it moves in the opposite direction of the underlying benchmark on each day.

The volatility of underlying market also affects the frequency and efficiency of rebalancing. A long or a short ETF written on an index presenting high volatile pricing

\footnotetext{
12 Proshares: "Rebalancing Leveraged and Inverse Fund Positions." Refer to the following link: http://www.proshares.com/resources/tools/ rebalancing_leveraged_or_inverse_fund_positions.html.
} 
behavior may require more frequent trades to rebalance than an ETF based on an index with lower volatility.

Finally, when trigger-based rebalancing techniques are employed, the frequency of rebalancing depends on the percentage threshold set. In particular, a large percentage trigger implies that fewer trades may be required over time. Therefore, a trigger of $\pm 10 \%$ will require less rebalancing than one of $\pm 5 \%$, albeit the size of the required trades may be larger.

\subsection{Performance illustration}

In regards of the leveraged ETFs' return, Table 1 provides a numerical example on how the performance of these products works. In particular, an investment of $\$ 1,000$ in a double-leveraged (bull) ETF is considered along with an equivalent investment in an inverse double-leveraged (bear) ETF. These ETFs have the same reference index, whose opening value on day 1 equals the 1,000 units.

\section{Table 1. Performance mechanism of leveraged and inverse ETFs}

\begin{tabular}{lcccc}
\hline \multicolumn{5}{c}{ Day 1 } \\
\hline Opening value & Return on day 1 & Closing value & Total return \\
\hline $2 \times$ Bull ETF & 1,000 & $10 \%$ & 1,100 & $+10 \%$ \\
\hline $2 \times$ Bear ETF & $\$ 1,000$ & $+20 \%$ & $\$ 1,200$ & $+20 \%$ \\
\hline & $\$ 1,000$ & $-20 \%$ & $\$ 800$ & $-20 \%$ \\
\hline Index & Opening value & Return on day 2 & Closing value & Total return \\
\hline $2 \times$ Bull ETF & 1,100 & $-10 \%$ & 990 & $-1 \%$ \\
\hline $2 \times$ Bear ETF & $\$ 1,200$ & $-20 \%$ & $\$ 960$ & $-4 \%$ \\
\hline
\end{tabular}

It is supposed that on the first day the underlying index achieves a $10 \%$ performance. This performance means that the value of the index at the end of trading day 1 reaches the 1,100 units. Going further, the bull and the bear ETFs are supposed to have performed on day 1 as they are designed to, namely the leveraged ETF gained a 20\% return while the inverse ETF made a $20 \%$ loss on its initial investment. The closing values of these ETFs on day 1 are $\$ 1,200$ for bull ETF and $\$ 800$ for bear ETF.

On day 2 , the index declines by $10 \%$ and its value at the end of day 2 equals the 990 units. Similarly to day 1 , the ETFs perform as they are designed to; the price of the bull ETF decreases by $20 \%$ and falls to $\$ 960$ and the value of bear ETF climbs to $\$ 960$ gaining a $20 \%$ return for this day. Compared to the initial values at the 
opening of day 1 , after two days of trading, the index has suffered an accumulated loss of $1 \%$ while both the bull and the bear ETFs lost $4 \%$ of their value at the beginning of day 1 .

This theoretical example accentuates the issue of compounding when assessing the long-term return of leveraged ETFs. More specifically, whereas the leveraged ETFs have achieved their goals on each single day, their two-day return differs from the daily stated multiples ( 2 and -2 times the index return for the bull ETF and the bear ETF, respectively). Interestingly, the compounded return of bear ETF does not even have the "correct" sign. Particularly, if compounding was not an issue, based on the cumulative loss of the index by $1 \%$, the bear ETF would be expected to gain a $2 \%$ return. On the contrary, this ETF has shriveled by $4 \%$ albeit its return on each day was the proper one.

An inference made from the analysis above is that leveraged and inverse ETFs are highly path dependent and the beyond one day performance offered to investors can be very hard to predict. The analysis also highlights the very short-term investment horizon of bull and bear ETFs. ${ }^{13}$ Moreover, it implies that investors should not be misled with respect to the usage of leveraged ETFs. This means that in no circumstances, are these products to be used in buy-and-hold investing strategies and, therefore, confusion about their performance objectives and frustration when their long-term return abstains from the performance of the underlying assets should not be expected on condition that the vendors of such ETFs do make the relevant warnings in the prospectuses of funds.

\subsection{Rebalancing and performance}

It has already pointed out that the constant rebalancing is necessary in order for the bull and bear ETFs to achieve their daily targets as well as that the compounding of daily returns usually results in leveraged ETFs underperforming or outperforming their benchmarks in the long-run. Little (2010) provides an enlightening analysis of leveraged ETFs' long-term under/out-performance compared to the traditional ETFs by discriminating between the static and dynamic leverage in a simple two-day example. Static was the leverage investors in the first-generation ETFs perceived to be obtaining. On the other hand, dynamic is the leverage available with the new leveraged ETFs.

The example assumes an investor who has one dollar available for investment and also borrows $(M-1)$ dollars. This investor totally invests $M$ dollars in a simple index

\footnotetext{
${ }^{13}$ An illustrative example of the extremely short-term horizon of leveraged ETFs is provided by Jason Sweig from the Wall Street Journal in the issue released on February 28, 2009. The columnist reports that on February 25, 2009, the trading volume for Direxion Financial Bear 3X totalled 23.I million shares on only two million shares outstanding. This figure implies an average holding period of less than 34 minutes. ("How Managing Risk with ETFs Can Backfire." Wall Street Journal, February 28, 2009.)
} 
ETF whose leverage is equal to unity. ${ }^{14}$ On the first and the second day, the underlying index produces $r_{1}$ and $r_{2}$ return, respectively. At the end of the second day, the $(M-1)$ debt must be repaid and the value of the static position is:

$$
\text { Value with Static Leverage }=M\left(1+r_{1}\right) \times\left(1+r_{2}\right)-(M-1)
$$

At the end of the second trading day, the index ETF has made an accumulated return amounting to $\left(1+r_{1}\right) \times\left(1+r_{2}\right)-1$ and the investor with the static leverage position has earned $M$ times the performance of the index. Had the investor invested their one dollar in a leveraged ETF written on the same index with a leverage multiple of $M$, they would have earned $M \times r_{1}$ on the first day and $M \times r_{2}$ on the second day. In this case, the value of the leveraged ETF applying daily rebalance would be:

$$
\text { Value with Leverage Rebalanced Daily }=1 \times\left(1+M \times r_{1}\right) \times\left(1+M \times r_{2}\right)
$$

The leveraged ETF has a cumulative two-day return of $\left(1+M \times r_{1}\right) \times\left(1+M \times r_{2}\right)$, which is different from the return of the static case providing that the $r_{1}$ and $r_{2}$ index returns are not nil. Subtracting equation 1 from equation 2 , we obtain the difference between the static and daily rebalanced leverage, which is:

$$
\text { Leverage }=\left(M^{2}-M\right) \times\left(r_{1} \times r_{2}\right)
$$

In equation 3 , the term $\left(M^{2}-M\right)$ is always positive given that the leverage of bull ETFs is equal to $+2 x$ or $+3 x$ and the leverage of bear ETFs is equal to $-1 x,-2 x$ or $-3 x$. Consequently, whether a leveraged ETF under- or out-performs its statically levered peer at the cumulative level depends on the term $\left(r_{1} \times r_{2}\right)$. In this respect, Little (2010) reports four possibilities.

The first possibility assumes that the price of the underlying index rises on both days. In this case, the term $\left(r_{1} \times r_{2}\right)$ is positive and the leveraged ETFs derives more than $M$ times the index return, which coincides with the return of the static leverage position. Thus, the dynamically leveraged ETF outperforms the statically leveraged one. The intuition is that rebalancing of leveraged ETF's exposure to index return at the end of first day is in favor of an advanced gain on the second day. In this case scenario, the effect of the dynamically rebalanced leverage on an inverse leveraged ETF will be that the bear ETF will lose less than if static leverage were employed.

The second possibility supposes that the return of the underlying index declines on both days, which also results in a positive $\left(r_{1} \times r_{2}\right)$. As a consequence, the leveraged

\footnotetext{
${ }^{14}$ It is implied that the performance of the simple index ETF equals index's return because it is considered before fees, expenses and taxes so that the analysis can focus on the impact of rebalancing.
} 
ETF applying daily rebalancing is found again to be in a better position than if a static leverage policy were adopted, that is, the leveraged ETF outperforms the static position.

The third alternative considers that the value of benchmark remains constant on both the two trading days. In this case, the term $\left(r_{1} \times r_{2}\right)$ is equal to zero and, thus, both the static and dynamic leverage positions derive nil performance.

The fourth and last possibility presumes that the index has non-nil returns of different sign on the first and the second day. In this case, the term $\left(r_{1} \times r_{2}\right)$ is negative and the leveraged ETF derives less than $M$ times the index return. As a result, the dynamic leverage causes the underperformance of leveraged ETF compared to the statically leveraged peer. Underperformance is explained via considering that if the index moves upwards on the first day, the exposure of leveraged ETF to the index will be increased due to rebalancing, resulting in a greater loss on day 2 in comparison to the static ETF. On the other hand, if the value of index goes down on the first day, the rebalancing of leveraged ETF will lower the exposure to the index and, thus, the gains on the second day will be inferior for leveraged ETF to the gains of the static peer.

\subsection{Volatility and performance}

The return of leveraged and inverse ETFs is crucially affected by the volatility in the underlying indices. Sullivan (2009) notes that the cumulative return achieved by leveraged ETFs is eroded by the variability in the prices of underlying assets. Moreover, the higher volatility over time, the lower the cumulative return and wealth to investors. He also notes that the long-run negative impact of market volatility on leveraged ETFs' returns worsens as the multiplier, (i.e. the leverage ratio), increases. On the contrary, even though the long-run performance of the ETF can be significantly lower than the stated multiple or even the raw return of the tracking index itself, the variance of ETF's returns can be as high as the index's variance or even greater. In summary, the ultimate effect of volatility is that investors in leveraged ETFs are exposed to at least the double of the market's risk without being compensated for this exposure by receiving twice the market return. Therefore, investors should always bear in mind that particularly in volatile markets they must monitor their investments in leveraged ETFs so as to ensure that their exposure to the underlying assets is in line with their desired objectives.

In Sullivan's (2009) analysis of volatility, it is pointed out that the selection of investment horizon is crucial in order for an investor in a leveraged ETF to receive the stated multiple over a long period. When the horizon is short, that is one day or less, the investor is highly likely to gain the stated multiple. However, if a longer interval is 
considered, the probability of investor to receive the stated return fades away due to greater unpredictability related to the volatility in the tracking index's pricing behavior.

The impact of volatility on the return of leveraged ETFs must be considered in addition to the impact of daily rebalancing by discriminating between dynamic and static leverage and considering a relevantly long-run investment horizon. In this respect, Little (2010) expands the analysis of the four possible outcomes described in the previous sub-section by suggesting additional four alternative returns resulting from the combined impact of rebalancing and volatility.

The first outcome concerns a market index moving upwards without significant fluctuations, that is, the volatility of the index is low. In this case, the leverage ETF returns more than $M$ times the performance of index. This outperformance is due to rebalancing levering up the gain on day 1 in advance of successive gains on the subsequent days. Therefore, in the case of an ascending and low volatile market, the dynamic rebalancing can beat the static leverage.

The second combination relates to a recessing market with low variability. Similarly to the first possibility, the leveraged ETF outperforms the statically leveraged peer. The reasoning behind outperformance is that in the declining market the leveraged ETF reduces its exposure to the underlying index and, consequently, loses less than $M$ times the index return. Again, as the fluctuation in index prices is trivial, the dynamic leverage strategy expressed by a leveraged ETF can beat the static one as it is depicted by the tracking index or the corresponding simple-beta ETF.

The third possibility assumes that the value index does not change over a long period but exhibits low volatility during this period. In this case, the leveraged ETF can return less than $M$ times the index return. Given that the return of the index is nil as a result of the index's price remaining unchanged, the return of the dynamically leveraged ETF will be negative while it would be expected to produce zero return. Therefore, in this scenario, the dynamic leverage is inferior to the static one.

The fourth alternative relates to a flat market, i.e. market with no clear trends which, however, presents high volatility. In this case, the combined impact of volatility and rebalancing can be materially harmful to long-run performance. In particular, the leveraged ETF actually follows a momentum strategy under which it increases exposure to the index on a profitable day and decreases exposure when market prices decline. This rebalancing activity is hard to perform in a market lacking in direction. If efficient active management by the managers of leveraged ETFs is absent too, the ETF will end up returning less $M$ times the return of the index and underperforming the static peer. 
Before concluding this section, it should be noted that the analysis of volatility and rebalancing's impact on performance has been made without considering the costs associated with the daily rebalancing as well as the relevant fees paid to the managing company of a leveraged ETF. In this respect, Little (2010) emphasizes the fact that these costs can negatively impact the cumulative return of leveraged ETFs even when markets are not highly volatile and they present favorable trends.

\section{Tax considerations}

The first-generation ETFs are considered to be tax efficient because their shares are redeemed in-kind via the exchange of the underlying securities. This mechanism results in a low portfolio turnover and limited or nil distribution of taxable capital gains. On the contrary, the in-kind redemption process is rather not the case for leveraged ETFs because their portfolio includes to a large extent futures, options and swaps and when a bull or a bear ETF is to be redeemed, the managing firm may be forced to liquidate some of the derivatives to raise the necessary cash to meet the redemptions. The liquidation of derivatives can result in the distribution of a taxable gain or loss for the remaining investors in the ETF. Moreover, the leveraged ETFs usually experience high portfolio turnover ratios as a result of the daily rebalancing in response to market movements. Therefore, the leveraged ETFs cannot be considered tax efficient.

Tax efficiency is a critical criterion in order for investors to decide on investing in leveraged ETFs or not. In general, the distributions of capital gains or losses are not welcomed by investors with a long-term horizon, who seek to delay the recognition of gains so that gains will compound through time before investors paying the respective taxes on these gains. Moreover, this kind of investors aims at benefiting from the lower tax rates on long-term capital gains. Thus, the leveraged ETFs would not be suitable investment vehicles for tax-averse long-term investors.

Contrary to the tax attitude of long-term investors, the leveraged ETFs are more appropriate for active asset allocators and short-term traders, who are less sensitive to distributions than the long-term investors. In general, the short-term traders do not seek to postpone the realization of capital gains or to defer the payment of the relevant taxes. Consequently, they allow short-term capital gains to accrue. These gains are treated as ordinary income for tax purposes and the short-term trader will be negatively affected from a tax perspective by the distribution only if the amount of distribution is greater than the trader's short-term capital gains in the current fiscal year. ${ }^{15}$

\footnotetext{
${ }^{15}$ This analysis of tax implications for a short-term trader with leveraged ETFs have been found on the website of Direxion Shares, which is one of the key companies providing and managing leveraged ETFs. Refer to: www.direxionfunds.com/literatures/ understanding-taxable-distributions. $\square$
} 
When it comes to capital losses, it should be noted that they cannot be used to offset distributions of income or capital gains, which are treated as ordinary income. As a consequence, a short-term trader having no short-term gains to compensate for losses realized upon the sale of their ETF shares within the year in which they received a distribution, they will be harmfully affected by the distribution. On the contrary, if the distribution concerns long-term capital gains, the losses from the sale of ETF shares mentioned above will be offset by these gains. Ultimately, there will be no negative tax impact on the short-term trader by the distribution.

The last tax issue concerns the suitable time for an investor to buy or redeem a leveraged ETF from a tax perspective. Usually, the most ETF providers (both of regular and leveraged ETFs) will distribute their annual gains or losses during the last quarter of the year. It is common practice for some ETF providers to announce tax distributions a few weeks in advance. However, other vendors make the relevant announcements only a few days before the distribution takes place. Therefore, investors need to monitor such announcements and adjust their investing decisions to their tax preferences. In particular, the holder of a leveraged ETF, which is expected to carry a large tax liability, will probably benefit from selling the ETF before the date of distribution. On the other hand, an investor, who examines whether to invest in the specific ETF, will probably be better off postponing the purchase until after the distribution date.

\section{Advantages and disadvantages of leveraged ETFs}

There are certain advantages offered to investors by the leveraged ETFs. As already noted, they are a simple way for investors to obtain leverage without needing to invest directly in derivatives or execute multiple costly trades. Avoiding of investment in derivatives further means that investors in leveraged ETFs do not need to maintain any margin account, which in turn means that investors do not need to refinance their position when the accumulated loss from the investment in the derivative has decreased the margin's amount at a level which is lower than the required one. ${ }^{16}$

Moreover, the leveraged ETFs are a flexible tool as they continuously trade throughout the day and when the prices move upwards, they do offer significant chances of investors cashing in on the current market trends. This is also the case

\footnotetext{
${ }^{16}$ This type of margin is called "variation" or "maintenance" margin and relates to a daily payment of profits and losses derived from the fluctuation in the value of a derivative such as a future contract. Futures are marked-to-market on a daily basis. In this process, the price of the contract on the current day is compared to the price on the previous day. The profit or loss on the day is then paid to or debited from the holder by the futures exchange, which acts as the counterparty to all contracts.
} 
within a declining market with an inverse leveraged ETF to be the suitable vehicle to make short-term gains.

Going further, the management expenses of leveraged ETFs are relevantly low as their average management expense ratios tend to be lower than those of the classic actively managed mutual funds. It should be noted however that the expense ratios of leveraged ETFs are significantly higher than the expense ratios of the regular ETFs or other passively managed investment tools such as index funds. The latter element is a disadvantage of leveraged ETFs from an expense-averse investor's perspective.

Another advantage of leveraged ETFs is that they can be used to lower the risk of an investor's portfolio. The mitigation of volatility is achieved by applying short selling techniques. However, these techniques are not recommended to the average unsophisticated investor, who may be unaware of the hazards relating to shortselling leveraged ETFs, such as the high possibility of losing the entire initial investment. Yet, investors going short leveraged ETF shares cannot lose more than the initial investment while when shorting ordinary stocks the magnitude of the possible loss is infinite. This is another advantage of leveraged ETFs over ordinary stocks in particular.

Apart from the important benefits of leveraged ETFs, they are some handicaps too. The most significant is that they cannot be used in buy-and-hold strategies. As already pointed out, for periods exceeding one day, an investor should not expect that they will earn two/three times the return of the tracking index due to the issues of daily rebalancing, the impact of underlying market's volatility and the compounding of returns. In addition, they are highly sensitive to market volatility and, thus, if returns fluctuate wildly over a relevantly long period, at the end of this period investors will probably have lost money even if the index has broken even. Other factors, fees and transaction costs included, can also make return deviate from the index's performance.

Another source of disadvantage relates to the exposure of leveraged ETFs to counterparty risk due to the employment of derivative products to deploy the required leverage. Even though there are certain ways to deal with the counterparty risk, investors cannot be sure that this risk will be absolutely tackled.

A last disadvantage for leveraged ETFs concerns some practical issues relating to short-selling as they are described by Elston and Choi (2009). At first, the broker may not find the necessary shares to perform short-selling. Second, the broker retains the right to terminate the short position anytime they wish. Finally, the accounting for of shorting for the investor may be difficult, costly and time consuming. 


\section{Leveraged versus traditional ETFs}

There are certain but not many similarities between the traditional ETFs and the leveraged ones. The main similarity between them is that they both are exchange traded products (ETPs) offering flexible trading throughout the entire day. Unlike open mutual funds, both the traditional and leveraged ETFs can be purchased or redeemed at any time during the trading day whereas mutual funds are traded only at the end of the day.

The second similarity relates to the variety in indices and markets tracked by the traditional and leveraged ETFs. In particular, the both types of ETF products provide investors with access to an extended row of assets including equities, fixed-income instruments, money and currency markets, commodities and precious metals. However, it must be noted that given the longer history of traditional ETFs, the variety of markets accessed via them is incomparably wider than that available with the leveraged ETFs.

Another and maybe the last material similarity between regular and leveraged ETFs is that they both can trade at a price which will abstain from the value of their underlying assets. However, the magnitude and the duration of premium or discount in the trading prices of these ETF types may vary depending on the arbitrage mechanism in place to correct the divergences between trading and net asset values. ${ }^{17}$

Contrary to the limited similarities, there are several differences between leveraged and first-generation ETFs. The first one relates to their investing objectives. The traditional ETFs seek to replicate the return of the selected indices. On the contrary, the leveraged ETFs actually aim at beating their benchmarks by providing double or triple the return of the indices. The outperformance goal is applicable both to leveraged and inverse ETFs, with the latter focusing on exploiting a declining market trend.

The second difference regards the investment horizon of these products. The leveraged ETFs aim at very short-term gains whose life does not exceed the one day. On the other hand, the traditional ETFs are mainly used by investors having long-term investing perspective. Given the extremely short-term investment horizon of leveraged ETFs, these products should not be considered as suitable vehicles for buy-and-hold investors, who expect long-term profits from their investments. Leveraged ETFs are probably better suited to sophisticated investors, such as the daily traders, because they are considered to be complex products and not easy for retail investors to fully perceive.

${ }^{17}$ It should be noted that NAV of leveraged ETFs is made known only at the end of day and, thus, in-cash arbitrage is available only at the end of the day or the beginning of the next trading day the latest (Shum, 20II). 
Another difference concerns the synthesis of portfolios. The majority of regular ETFs are usually fully invested in the whole range of stocks comprising the underlying index and at the same weightings in order to obtain the desired exposure to the index and enhance the replication of its return (physical replication). On the contrary, a leveraged ETF may include the equities comprising the index but also derivatives on these stocks or the index (synthetic replication). This difference is critical because it entails different kinds of risks and liabilities between the two ETF types.

Maybe the most important difference relates to leverage and the rebalancing of portfolio on a daily basis. A single-beta ETF is considered to seek a 1:1 leverage with the underlying index. On the other hand, a leveraged ETF is designed to double or triple leverage in a positive or a negative fashion. In addition, the portfolio of a leveraged ETF is rebalanced on a daily basis in response to market volatility and changes in the value of the tracking index in order for the designed leverage to be maintained. On the contrary, the portfolio of a classic ETF is occasionally rebalanced and only in response to changes in the synthesis of the index. The main implication of this is that, in the long-run, the performance of leverage ETFs is likely to significantly deviate from the long-run return of their corresponding benchmarks whereas the longterm performance of a traditional ETF can be considered a sufficient approximation of index's return. ${ }^{18}$

Going further, there is a difference between the costs charged to investors by classic and leveraged ETFs, respectively. In general, it is a fact that the latter are more expensive than the former. ${ }^{19}$ The expense "superiority" is due to the more frequent transactions a leverage ETF is obliged to perform than a regular ETF, the financial and borrowing cost relating to the indebtedness involved in its working mechanism and its more active management along with the consequent higher fees paid for this.

The last material difference between leveraged and classic ETFs emanates from the different level of tax efficiency they offer to their investors. As it has already been explained in a previous section, the regular ETFs are actually considered as a high taxefficient investing tool due to the limited distributions of taxable capital gains or losses, the low portfolio turnover and the unique in-kind redemption of their shares. On the contrary, the redemption of leveraged ETF shares is executed in cash, the turnover is higher and they are subject to more frequent distributions. As a consequence, the tax efficiency of leveraged ETFs is not that remarkable as for traditional ETFs.

\footnotetext{
${ }^{18}$ The return of classic ETFs is certainly subject to the tracking error issue but tracking error is usually lower for these ETFs compared to leveraged ETFs.

${ }^{19}$ In this respect, Rompotis (20I I), who examines the leveraged versus the regular ETFs, reports an average expense ratio for leveraged and comparable regular ETFs of $0.94 \%$ and $0.33 \%$, respectively.
} 


\section{References}

Avellaneda, M. and Zhang, S.J. (2010). Path-Dependence of Leveraged ETF Returns, SIAM Journal on Financial Mathematics, 1(1), pp. 586-603.

Avellaneda, M., and Zhang, S.J. (2010). Leveraged ETFs: All You Wanted to Know but Were Afraid to Ask, Risk Professional, February, pp. 54-60.

Barnhorst, B.C. and Cocozza, C.R. (2011). Inverse and Leveraged ETFs: Considering the Alternatives, Journal of Financial Planning, 24(1), pp. 44-52.

Bai, Q., Bond, S.A. and Hatch, B. (2012). The Impact of Leveraged and Inverse ETFs on the Underlying Stock Returns, Working Paper, University of Cincinnati.

Blau, B.M. and Brough, T.J. (2011). Is the Trading of Inverse ETFs a Bearish Signal?, Journal of Trading, 6(3), pp. 32-40.

Bush, M. (2009). Gearing Up For Leverage: An In-Depth Review of a Growing Market Phenomenon. Available on: www.indexuniverse.com $\square$

Carver, A.B. (2009). Do Leveraged and Inverse ETFs Converge to Zero?, ETF and Indexing, Institutional Investors Journals Investment Guides, 1, pp. 144-149.

Charupat, N. and Miu, P. (2011). The Pricing and Performance of leveraged Exchange-Traded Funds, Journal of Banking and Finance, 35(4), pp. 966-977.

Chen, J-H. and Diaz, J.F. (2012). Spillover and Asymmetric-Volatility Effects of Leveraged and Inverse Leveraged Exchange Traded Funds, Journal of Business and Policy Research, 7(3), pp. 1-10.

Cheng, M. and Madhavan, A. (2009). The Dynamics of Leveraged and Inverse Exchange-Traded Funds, Journal of Investment Management, 7(4), pp. 43-62.

Curcio, R.J., Anderson, R.I. and Guirguis, H. (2012). Should Tracking Error Prevent the Use of Leveraged ETFs in the Real Estate Portfolio? Journal of Index Investing, 3(3), pp. 75-95.

Dulaney, T., Husson, T. and McCann C. (2012). Leveraged, Inverse, and Futures-Based ETFs, Piaba Bar Journal, 19

Elston, F. and Choi, D. (2009). Inverse ETFs, Proceedings of the Allied Academies International Conference, 14(1), pp. 5-9.

- Guedj, I., Li, G. and McCann, C. (2010). Leveraged ETFs, Holding Periods and Investment Shortfalls, Journal of Index Investing, 1(3), pp. 45-57.

Haryanto, E., Rodier, A., Shum, P. and Hejazi, W. (2012). Intraday Share Price Volatility and Leveraged ETF Rebalancing. Available at SSRN: http://ssrn.com/abstract=2161057 or http://dx.doi.org/10.2139/ssrn.2161057 $\square$

Hill, J. and Foster, G. (2009). Understanding Returns of Leveraged and Inverse Funds, Journal of Indices, 12(5), pp. 40-58.

Hill, J. and Teller, S. (2009). Rebalancing Leveraged and Inverse Funds, ETF and Indexing, Institutional Investors Journals Investment Guides, 1, pp. 66-76. 
Hill, J. and Teller, S. (2010). Hedging with Inverse ETFs, Journal of Indices, 13(6), pp. 18-26.

Hougan, M. (2009). How Long Can You Hold Leveraged ETFs?, Journal of Indices, 12(2), pp. 36-41.

Leung, T. and Santoli, M. (2012). Leveraged Exchange Traded Funds: Admissible Leverage and Risk Horizon, Journal of Investment Strategies, 2(1), pp. 39-61.

Little, P.K. (2010). Inverse and Leveraged ETFs: Not Your Father's ETF, Journal of Index Investing, 1(1), pp. 83-89.

Lu, L., Wang, J. and Zhang, G. (2012). Long Term Performance of Leveraged ETFs, Financial Services Review, 21(1), pp. $63-80$

Mackintosh, P. (2008). Double Trouble, ETF and Indexing, Institutional Investors Journals Investment Guides, 1, pp. 25-31.

Murphy, R. and Wright, C. (2010). An Empirical Investigation of Commodity-Based Leveraged ETFs. Journal of Index Investing, 1(3), pp. 14-23.

Rollenhagen, K.J. (2009). Pitfalls and Potential: An Attempt to Demystify Leveraged and Inverse Exchange-Traded Index Funds, ETF and Indexing, Institutional Investors Journals Investment Guides, 1, pp. 105-107.

Rompotis, G.G. (2011). A Study on the 3G Exchange Traded Funds: The Case of Short ETFs, Journal of Index Investing, 2(1), pp. 25-43.

Rompotis, G.G. (2012). A Survey on Leveraged and Inverse Exchange Traded Funds, Journal of Index Investing, 2(4), pp. 84-95.

Rompotis, G.G. (2013). A Revised Survey on Leveraged and Inverse Exchange Traded Funds, Journal of Index Investing, 4(2), pp. 45-66.

nullivan, R. (2009). The Pitfalls of Leveraged and Inverse ETFs, CFA Magazine, May-June Issue, p. 12.

Shum, P. (2011). The Long and Shorts of Leveraged ETFs: the Financial Crisis and Performance Attribution, Working Paper, York University.

- Trainor, W.J. Jr. and Baryla, E.A. Jr. (2008). Leveraged ETFs: A Risky Double that Does not Multiply by Two. Journal of Financial Planning, 21(5), pp. 48-55.

Trainor, W.J. Jr. (2010) Do Leveraged ETFs Increase Volatility?, Technology and Investment, 1, pp. 215-220.

Trainor, W.J. Jr. (2011a). Daily vs. Monthly Rebalanced Leveraged Funds, Journal of Finance and Accountancy, 6 (May issue), pp. 1-14. Available on: http://www.aabri.com/jfa.html $\square$

Trainor, W.J. Jr. (2011b). Solving the Leveraged ETF Compounding Problem, Journal of Index Investing, 1(4), pp. 66-74.

Wang, Z. (2009). Market Efficiency of Leveraged ETFs, Working Paper, Federal Bank of New York.

Welker, A. (2012). Leveraged ETFs and Intraday Volatility, All Graduate Reports and Creative Projects. Paper 205. Available on: http://digitalcommons.usu.edu/gradreports/205 $\square$ 
